\title{
X-ray flares in the early Swift observations of the possible naked gamma-ray burst 050421
}

\author{
O. Godet ${ }^{1}$, K. L. Page ${ }^{1}$, J. P. Osborne ${ }^{1}$, P. T. O’ Brien ${ }^{1}$, D. N. Burrows ${ }^{1}$, J. E. Hill ${ }^{6,9}$, B. Zhang ${ }^{8}$, \\ A. P. Beardmore ${ }^{1}$, L. Angelini ${ }^{6}$, M. Capalbi ${ }^{3}$, J. Cummings ${ }^{6,7}$, N. Gehrels ${ }^{6}$, M. R. Goad ${ }^{1}$, J. A. Kennea ${ }^{2}$, \\ V. Mangano ${ }^{4}$, A. Moretti ${ }^{5}$, and D. C. Morris ${ }^{2}$ \\ 1 X-ray and Observational Astronomy Group, Department of Physics \& Astronomy, University of Leicester, LE1 7RH, UK \\ e-mail: og19@star.le.ac.uk \\ 2 Department of Astronomy \& Astrophysics, 525 Davey Lab, Pennsylvania State University, University Park, PA 16802, USA \\ 3 ASI Science Data Center, via G. Galilei, 00044 Frascati, Italy \\ 4 INAF - Istituto di Astrofisica Spaziale e Fisica Cosmica Sezione di Palermo, Via U. La Malfa 153, 90146 Palermo, Italy \\ INAF - Osservatorio Astronomico di Brera, via E. Bianchi 46, 23807, Merate (LC), Italy \\ 6 Goddard Space Flight Center, Greenbelt, Maryland 20771, USA \\ 7 National Research Council, 2101 Constitution Ave NW, Washington, DC 20418, USA \\ 8 Department of Physics, University of Nevada, Box 454002, Las Vegas, NV 89154-4002, USA \\ 9 Universities Space Research Association, 10211 Wincopin Circle, Suite 500, Columbia, MD, 21044-3432, USA
}

Received 28 October 2005 / Accepted 26 February 2006

\section{ABSTRACT}

\begin{abstract}
We present the Swift observations of the faint burst GRB 050421. The X-ray light-curve shows at least two flares: the first flare peaking at $\sim 110 \mathrm{~s}$ after the BAT trigger $\left(T_{0}\right)$ and the second one peaking at $\sim 154 \mathrm{~s}$. The first flare presents a flux variation of $\delta F / F_{\text {peak }} \sim 3.7$ and a short timescale ratio $\delta t / t_{\text {peak }} \sim 0.07$. The second flare is smaller and presents a flux variation of $\delta F / F_{\text {peak }} \sim 1.7$ and a short timescale ratio $\delta t / t_{\text {peak }} \sim 0.03$. We argue that the mechanism producing these flares is probably late internal shocks. The X-ray light-curve is consistent with a rapid decline with a temporal index $\alpha \sim 3.1$, which decays from $\sim 10^{-9} \mathrm{erg} \mathrm{cm}^{-2} \mathrm{~s}^{-1}$ at $T_{0}+100 \mathrm{~s}$ to $<7 \times 10^{-13} \mathrm{erg} \mathrm{cm}^{-2} \mathrm{~s}^{-1}$ at $T_{0}+900 \mathrm{~s}$. A possible spectral softening is also observed with time, from $\beta \sim 0.1$ to $\sim 1.2$. A good joint fit to the BAT and XRT spectra before $T_{0}+171 \mathrm{~s}$ with $\beta_{\text {XRT-BAT }} \sim 0.2$ indicates that the early X-ray and Gamma-ray emissions are likely produced by the same mechanism. We argue that the X-ray spectral softening, if any, is due to a shift of the peak of the prompt emission spectrum down to lower energies, and that the rapid decline of the X-ray emission is probably the tail of the prompt emission. This suggests that the X-ray emission is completely dominated by high latitude radiation and the external shock, if any, is extremely faint and below the detection threshold. GRB 050421 is likely the first "naked burst" detected by Swift.
\end{abstract}

Key words. gamma ray: bursts

\section{Introduction}

The Swift Gamma-Ray Burst (GRB) Explorer was launched on 20th November 2004 (Gehrels et al. 2004). It is a multiwavelength observatory covering the Gamma-ray, X-ray and UV/optical bands. After the detection of a GRB by the Burst Alert Telescope (BAT) (Barthelmy et al. 2005), the observatory slews automatically and rapidly to point the narrow field instruments: the X-ray Telescope (XRT, Burrows et al. 2005), and the UV/Optical Telescope (UVOT, Roming et al. 2005). Due to its unique capability to slew on a timescale of minutes and its high sensitivity, Swift is ideal to study the properties of the early afterglow, in particular the transition between the prompt emission and the afterglow.

The current Gamma-Ray Burst picture is that the GRB and the afterglow are produced by a relativistically expanding blastwave associated with internal and external shocks (Mészáros \& Rees 1993; Rees \& Mészáros 1994; Kobayashi et al. 1997; Panaitescu \& Mészáros 1998). In the simplest version of this model, the prompt Gamma-ray emission is produced by internal shocks. The duration of the prompt emission extends from
$0.01 \mathrm{~s}$ to $1000 \mathrm{~s}$ with two distinct GRB populations: the $<2 \mathrm{~s}$ short GRBs peaking typically at $\sim 0.2 \mathrm{~s}$ and the $<2$ s long GRBs peaking typically at $\sim 20 \mathrm{~s}$ (e.g. Kouveliotou et al. 1993; Dezalay et al. 1996; Paciesas et al. 1999).

Currently, it is not completely understood how the central engine can still be active over a timescale extending from $0.01 \mathrm{~s}$ to $1000 \mathrm{~s}$ for the long GRBs. Sari \& Piran (1997) have shown that the time structure of GRBs must be intrinsic to their energy source. A possible explanation marking a departure from the current hypernova picture is that the extended activity of the GRBs results in the fragmentation of the collapsing stellar core in proto neutron stars and the subsequent merger of these fragments (Davies et al. 2002; King et al. 2005). Other models have also been proposed (e.g. see Mészáros 2000; Zhang \& Mészáros 2004, and references in these papers).

When the blastwave shocks the surrounding medium, it is decelerated and a broad-band afterglow is produced. The afterglow emission usually fades with time as a broken power-law. For a more detailed picture of this model, see e.g. Zhang \& Mészáros (2004). 
Recent X-ray observations of GRBs 100-1000 s after the trigger have shown unexpected and complex light-curve behaviour, in particular a steep-to-flat transition in the early temporal decay (e.g. Tagliaferri et al. 2005; Burrows et al. 2005b; Nousek et al. 2006). An explanation of the initial steep decline of the X-ray light-curve is perhaps the curvature effect (Kumar \& Panaitescu 2000; Zhang et al. 2006). This results in delayed arrival of the prompt emission from the high angular latitude regions of the blastwave relative to the observer direction.

$\mathrm{X}$-ray flares have also been reported in the early X-ray lightcurves. XRF 050406 was the first Swift GRB for which such a feature was observed by the XRT (Romano et al. 2006), peaking at $\sim 210 \mathrm{~s}$ after the trigger. GRB $050502 \mathrm{~b}$ also presented an X-ray flare peaking at $345 \mathrm{~s}$ after the trigger, but with the fluence of the flare larger in the XRT band than in the prompt Gamma-ray emission itself in the 15-350 keV band (Burrows et al. 2005b; King et al. 2005; Falcone et al. 2006). Moreover, smaller flares could be seen for this burst beyond $\sim 10^{4}$ s. Other Swift GRBs also show X-ray flares in their light-curve (e.g. Nousek et al. 2006; O'Brien et al. 2006). Most of the flares seen in the X-ray light-curves have been interpreted as evidence that the internal shock activity extends beyond the prompt Gamma-ray emission.

Piro et al. (2005) also reported the observation of X-ray flares in GRB 011121 and GRB 011211. The authors have argued that the late X-ray flares represent the onset of the afterglow, because the spectral slopes of the flares are inconsistent with those of the prompt emission, but consistent with those of the afterglow. In addition, they have shown that the tail of the X-ray flares and the light-curve of the afterglow at 1 day are connected by a single power law $\sim\left(t-t_{0}\right)^{-\alpha}, t_{0}$ corresponding to the onset of the X-ray flares.

We report in this paper the Swift observation of the GRB discovered by the BAT on 21st April 2005. The observatory automatically slewed to the BAT position. The XRT onboard software found and centroided on a rapidly decaying X-ray source. This source exhibited two X-ray flares in its light-curve and, at $T_{0}+900 \mathrm{~s}$, became undetectable (with an $3 \sigma$ upper limit on the unabsorbed X-ray flux $F_{v}<7 \times 10^{-13} \mathrm{erg} \mathrm{cm}^{-2} \mathrm{~s}^{-1}$ in the $0.3-10 \mathrm{keV}$ band).

The paper is organised as follows: in Sect. 2, we present the characteristics of the observations and the basic steps of the data reduction. In Sect. 3, we present the spectral and temporal analysis of the multi-wavelength observations. In Sect. 4, we investigate the mechanisms producing the spectral and temporal characteristics of the burst. We also explore the possibility that the flares in the light-curve could be the evidence of late internal shocks.

By convention, we note hereafter the flux in the X-ray band is modelled as $F_{v} \propto v^{-\beta} t^{-\alpha}$ where $\beta$ is the energy spectral index and $\alpha$ is the temporal index. We use the symbol $\Gamma$ to refer to the Lorentz factor. The BAT spectral slope is noted as $\beta_{\text {BAT }}$ and $T_{0}$ is the trigger time.

\section{Observation and data reduction}

\subsection{BAT observations}

GRB 050421 (Swift-BAT trigger 115135) was detected at 04:11:52 UT on 21st April 2005 at $(\mathrm{J} 2000) \mathrm{RA}=20^{\mathrm{h}} 28^{\mathrm{m}} 58^{\mathrm{s}}$ and Dec $=+73^{\circ} 39^{\prime} 54^{\prime \prime}$, with an uncertainty of 4 arcmin (Barbier et al. 2005).

The BAT spectra and light-curves were extracted using the BAT analysis software (build 2.0) as described in the Swift BAT Ground Analysis Software Manual (Krimm et al. 2004).

\subsection{XRT observations}

The XRT started to observe $89 \mathrm{~s}$ after the trigger, following its normal sequence of readout modes: Low rate Photo-diode mode (LrPD) during the slew, Image mode (IM) when the spacecraft settled, Piled-Up Photo-Diode (PuPD), LrPD, Windowed Timing (WT), and then Photon Counting (PC) modes while pointed at the target and according to the source brightness (see Hill et al. 2004, 2005b). An uncatalogued X-ray source was identified on ground at $(\mathrm{J} 2000) \mathrm{RA}=20^{\mathrm{h}} 29^{\mathrm{m}} 01.7^{\mathrm{s}}$ and Dec $=+73^{\circ} 39^{\prime} 17.4^{\prime \prime}$ with an uncertainty of 5 arcsec at a $90 \%$ confidence level (Godet et al. 2005). Recent calibration of the XRT boresight has allowed an improvement in the XRT position measurements (Moretti et al. 2006). Accounting for this, the refined position is $(\mathrm{J} 2000) \mathrm{RA}=20^{\mathrm{h}} 29^{\mathrm{m}} 02.44^{\mathrm{s}}$ and Dec $=$ $+73^{\circ} 39^{\prime} 17.8^{\prime \prime}$ with a total uncertainty radius of 3.7 arcsec at a $90 \%$ confidence level.

The XRT data were processed by the Swift software version $2.0^{1}$. A cleaned event list was generated using the default pipeline, which removes the effects of hot pixels and the bright Earth.

From the cleaned event list, the source and background spectra were extracted using xselect. For the spectral analysis, only the WT and PC data were useful (see Sect. 3.2.2). The first $120 \mathrm{~s}$ of the PC data have a count rate above $1 \mathrm{cts} \mathrm{s}^{-1}$ and therefore, are slightly piled-up (see Vaughan et al. 2005, for a detailled discussion about the pile-up effect). The innermost 3 pixel radius was excluded, and the source and background spectra were extracted using an annular region with an outer 30 pixel radius. The later PC data were extracted using a circular region within a 30 pixel radius. For the spectral analysis, we used the grade 0 events for the PC and WT modes, for which the response matrices are presently the best calibrated. The ancillary response files for the PC and WT modes were created using xrtmkarf.

The light-curves were also extracted from the cleaned event list using xselect over the $0.3-10 \mathrm{keV}$ band. The LrPD grades $0-5$, PC grades 0-12 and WT grades $0-2$ events were used for the light-curves, giving slightly higher effective area at higher energies. The XRT observations are summarised in Table 1.

\subsection{UVOT observations}

The UVOT, which began to observe $112 \mathrm{~s}$ after the trigger, detected no optical fading source down to a $5 \sigma$ limiting magnitude of 17.5 in $V$-band, 19.1 in $B$-band and 18.8 in $U$-band, using coadded images of the early time data of $9 \times 10 \mathrm{~s}$ exposure (Blustin et al. 2005).

\subsection{Other observations}

No optical or IR transient has been detected. ROTSE-III (Rykoff et al. 2005) started to observe $67.2 \mathrm{~s}$ after the trigger, but no source was detected down to an unfiltered magnitude of 17.8 in $69 \mathrm{~s}$. The BOOTES-2 (Burst Observer and Optical Transient Exploring System) very wide field camera started to observe $8 \mathrm{~s}$ after the onset of the burst, and no prompt optical flash arising from this burst was detected with a limiting (unfiltered) magnitude of 9 (Jelinek et al. 2005). The $\sim 2000$ s observations starting at 11:23:45 UT on the Mount Hopkins in the $J, H$ and $K$ bands did not reveal any new sources detected above $3 \sigma$ within the XRT error circle (Bloom et al. 2005).

\footnotetext{
${ }^{1}$ Seehttp://heasarc.gsfc.nasa.gov/docs/swift/analysis/
} 
Table 1. Log of the XRT observations for GRB 050421 following the XRT mode sequence.

\begin{tabular}{cccccc}
\hline \hline Sequence & XRT mode & $\begin{array}{c}\text { Start time } \\
\text { (yy-mm-dd hh:mm:ss) }\end{array}$ & $\begin{array}{c}\text { End time } \\
\text { (yy-mm-dd hh:mm:ss) }\end{array}$ & $\begin{array}{c}\text { Start time since trigger }_{(\mathrm{s})} \\
\text { Exposure }^{a} \\
(\mathrm{~s})\end{array}$ \\
\hline 00115135000 & LrPD slew & $2005-04-2104: 13: 21$ & $2005-04-2104: 13: 28$ & 89 & 7.2 \\
00115135000 & LrPD settling & $2005-04-2104: 13: 29$ & $2005-04-2104: 13: 37$ & 97 & 8.2 \\
00115135000 & IM & $2005-04-2104: 13: 39$ & $2005-04-2104: 13: 41$ & 107 & 2.5 \\
00115135000 & PuPD & $2005-04-2104: 13: 44$ & $2005-04-2104: 13: 45$ & 112 & 1.3 \\
00115135000 & LrPD pointing & $2005-04-2104: 13: 45$ & $2005-04-2104: 13: 46$ & 113 & 1.4 \\
00115135000 & WT & $2005-04-2104: 13: 47$ & $2005-04-2104: 14: 43$ & 115 & 56 \\
00115135000 & PC & $2005-04-2104: 14: 43$ & $2005-04-2207: 53: 30$ & 171 & 34416 \\
00115135001 & PC & $2005-04-2300: 58: 12$ & $2005-04-2515: 41: 56$ & 161180 & 4008 \\
00115135002 & PC & $2005-04-2601: 16: 10$ & $2005-04-2619: 14: 57$ & 421546 & 6927 \\
\hline
\end{tabular}

${ }^{a}$ Only the exposure time for the PC mode is spread over several snapshots during each observation.

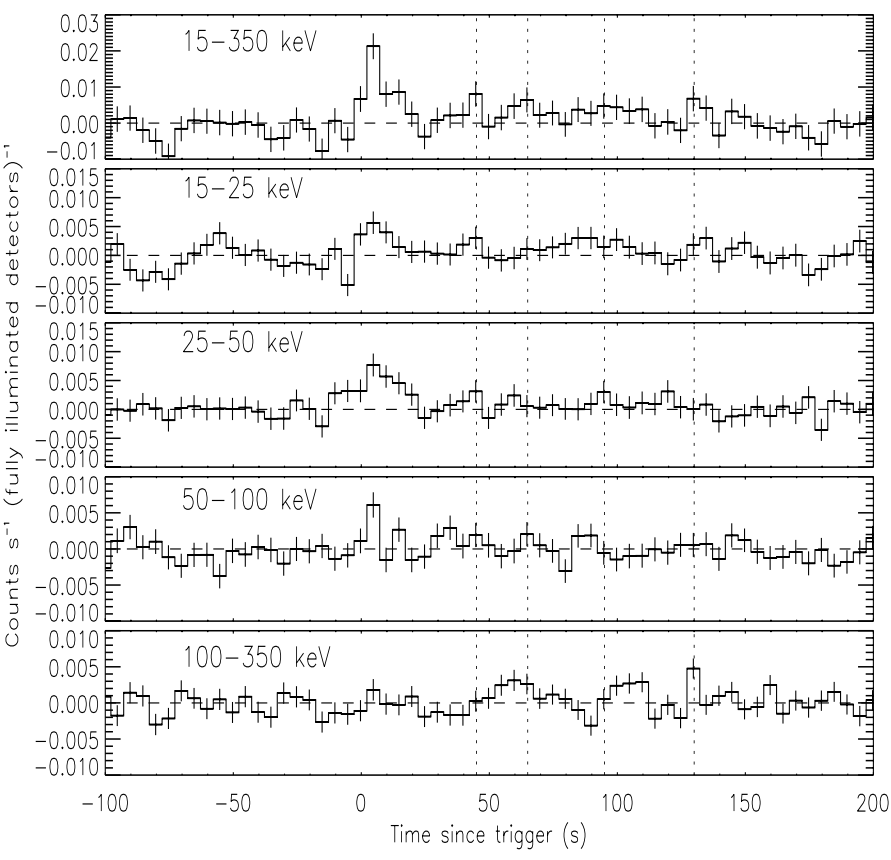

Fig. 1. BAT light-curve for 4 different energy bands from the top to the bottom: $15-350 \mathrm{keV}, 15-25 \mathrm{keV}, 25-50 \mathrm{keV}, 50-100 \mathrm{keV}$ and $100-350 \mathrm{keV}$. The dashed vertical lines represent possible smaller flares at $\sim 45, \sim 65, \sim 95-110$ and $\sim 130 \mathrm{~s}$ after the trigger. The BAT light-curve is given in units of counts $\mathrm{s}^{-1}$ (fully illuminated detector) ${ }^{-1}$.

\section{Data analysis results}

All the errors cited below are given at a $90 \%$ confidence level for one parameter of interest (i.e. $\Delta \chi^{2}=2.706$ ).

\subsection{Light-curve}

\subsubsection{Gamma-ray band}

The BAT light-curve of GRB 050421 has a Fast-Rise and Exponential-Decay (FRED) structure (Sakamoto et al. 2005), with a rise time less than $1 \mathrm{~s}$ and a decay time of $\sim 10 \mathrm{~s}$ (the upper panel in Fig. 1). Note that a tail or/and some further smaller flares may be present in the BAT light-curve. Figure 1 also shows the BAT light-curve in four different spectral bands.

In the $15-350 \mathrm{keV}$ band, $T_{50}$ and $T_{90}$ are $4.8 \pm 0.5 \mathrm{~s}$ and $10.3 \pm$ $0.3 \mathrm{~s}$ respectively, while the 1 -second peak photon flux is $0.5 \pm$ $0.1 \mathrm{ph} \mathrm{cm}^{-2} \mathrm{~s}^{-1}$. The fluence in the $15-150 \mathrm{keV}$ band measured from $T_{0}$ to $T_{0}+T_{90}$ is $1.1 \pm 0.7 \times 10^{-7} \mathrm{erg} \mathrm{cm}^{-2}$.

\subsubsection{X-ray band}

The X-ray light-curve of GRB 050421 over the $0.3-10 \mathrm{keV}$ band as shown in Fig. 2 combines data taken in all five data modes mentioned above. The light-curve from $T_{0}+97 \mathrm{~s}$ to $T_{0}+200000 \mathrm{~s}$ is fit using the BAT trigger time as the zero time.

The light-curve can be described by a simple and unbroken power law with a time decay index of $\alpha=3.1 \pm 0.1$ with at least two flares superposed $\left(\chi^{2}=27.6 / 28\right.$ d.o.f.). The two flares are fit by Gaussians. A possible third flare can be seen around $T_{0}+206 \mathrm{~s}$. The X-ray emission declines so rapidly that the X-ray emission became undetectable less than an hour after the trigger.

The index of the early steep decay is close to the mean of the observed range of X-ray temporal decay slopes given in Nousek et al. (2006) $\left(\alpha_{\text {mean }} \sim 3.4\right)$. Note that if we only fit the XRT lightcurve before $T_{0}+180 \mathrm{~s}$ with the same model as discussed above, we find a shallower temporal slope of $\alpha=2.0 \pm 0.6$.

The data for the first flare are sparse, because the flare occured at the end of the spacecraft settling phase. The flare was then observed through a sequence of four modes (the LrPD mode during the settling of the spacecraft on the source, and then the IM, PuPD and LrPD modes when the XRT was pointed at the source). Therefore, the temporal characteristics of the flare given below should be taken with caution. The best fit indicates that the first flare peaks at $T_{0}+110 \pm 2 \mathrm{~s}$ and has a fast rise with a small $\delta t_{\text {rise }} / t_{\text {peak }} \sim 0.05$. The timescale ratio for the decaying part of the flare $\delta t_{\text {decay }} / t_{\text {peak }}$ is $\sim 0.03$, where $\delta t_{\text {rise }}$ and $\delta t_{\text {decay }}$ are the time intervals respectively from the underlying power-law decay to the peak and from the peak to the underlying power-law decay. If the underlying power-law decay is subtracted, the rise is fit as $t^{9.7 \pm 1.2}$ from $T_{0}+97 \mathrm{~s}$ to $T_{0}+108 \mathrm{~s}$ and the decay as $t^{-\alpha}$ with $\alpha<10.7$ from $T_{0}+110 \mathrm{~s}$ to $T_{0}+118 \mathrm{~s}$. During the flare, the source re-brightens by a factor $\delta F / F \sim 3.7$ between $T_{0}+100 \mathrm{~s}$ and the peak.

The second flare is weaker, peaking at $T_{0}+154 \pm 3 \mathrm{~s}$ with $\delta t_{\text {rise }} / t_{\text {peak }} \sim 0.03\left(\delta t_{\text {decay }} / t_{\text {peak }} \sim 0.03\right)$ and $\delta F / F \sim 1.7$ measured between $T_{0}+144 \mathrm{~s}$ and the peak.

These two flares show values of $\delta t / t \ll 1$, which puts strong constraints on the physical mechanisms producing them (see Sect. 4.3).

\subsection{Spectroscopy}

\subsubsection{Gamma-ray band}

The pre-slew spectrum from $T_{0}-10 \mathrm{~s}$ to $T_{0}+T_{90}$ is well fit by a single power-law with the spectral index $\beta_{\mathrm{BAT}}=0.7 \pm 0.5$ (see Table 2), consistent with that given by Sakamoto et al. (2005). 


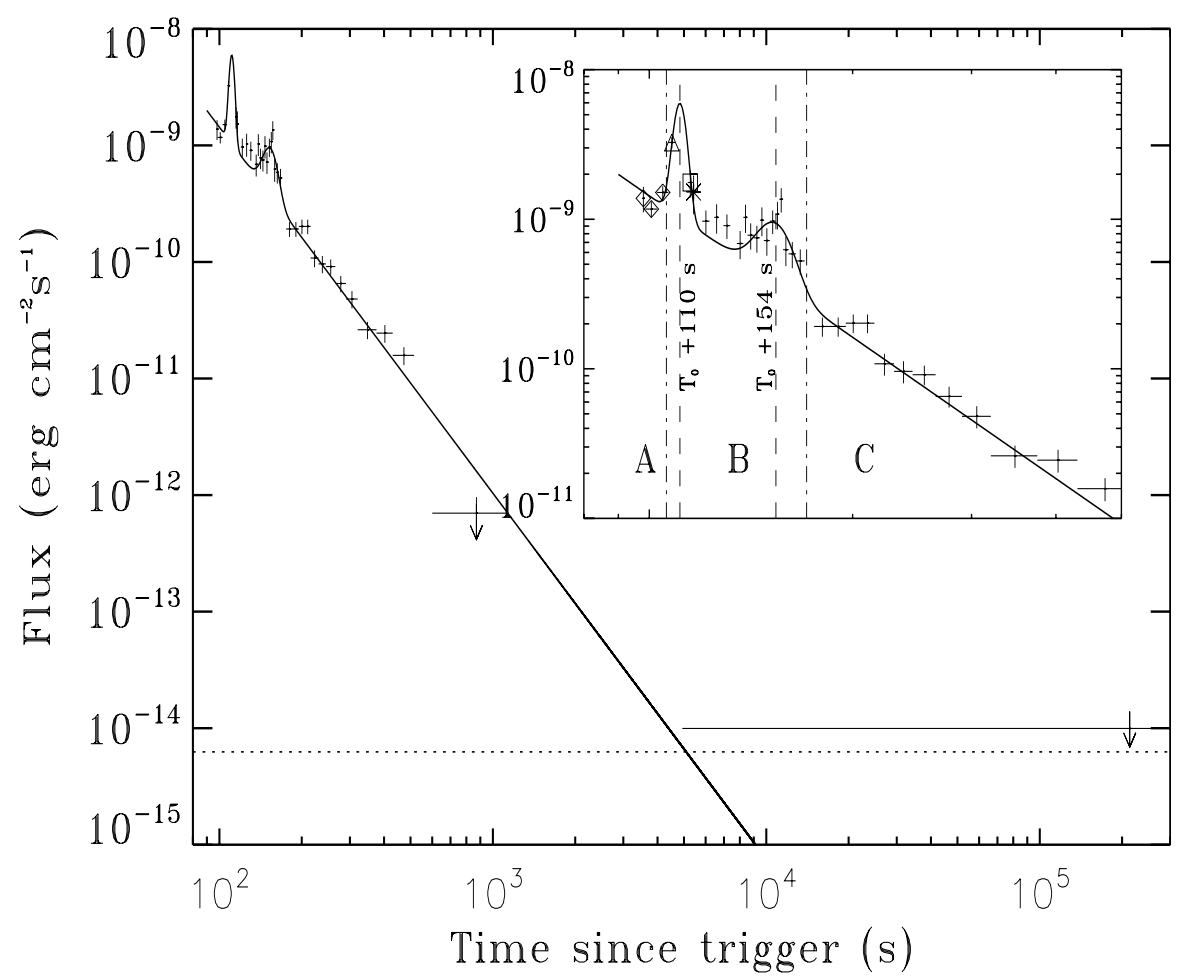

Fig. 2. Background subtracted XRT light-curve of GRB 050421 over the energy band 0.3-10 keV: A) LrPD slew and settling data (diamonds); B) IM (triangle), PuPD (square), LrPD (star) and WT pointing data; C) PC pointing data. The best fit to the light-curve is plotted by the solid line. The dotted line corresponds to the background level in the PC data. The upper limits are given at $3 \sigma$. The times of the two X-ray flares are also shown in the plot.

Use of a Band function (Band et al. 1993) or a cutoff powerlaw function did not significantly improve the fit. Swift GRB 050421 is the 44th faintest burst, in term of fluence, in a sample of 49 GRBs detected by the BAT ${ }^{2}$ before the end of August 2005. We also extracted a spectrum from $T_{0}+20 \mathrm{~s}$ to $T_{0}+100 \mathrm{~s}$ to investigate a possible spectral softening of the prompt emission in the GRB tail. However, no significant spectral softening is seen in the spectrum, since the spectral slope for the spectrum from $T_{0}+20 \mathrm{~s}$ to $T_{0}+100 \mathrm{~s}$ is $0.8 \pm 1.6$.

\subsubsection{X-ray band}

In the following analysis, we assume a Galactic absorption of $N_{\mathrm{H}}^{\mathrm{Gal}}=1.44 \times 10^{21} \mathrm{~cm}^{-2}$ (Dickey \& Lockman 1990). The WT spectrum covers the temporal range from $T_{0}+115 \mathrm{~s}$ to $T_{0}+171 \mathrm{~s}$, i.e. an exposure of $56 \mathrm{~s}$. The PC data were divided into two spectra. We denote the first $120 \mathrm{~s}$, from 171-291 s after $T_{0}$, as "early" and the $480 \mathrm{~s}$ of data from $291-771 \mathrm{~s}$ after $T_{0}$, as "late". Beyond $T_{0}+771 \mathrm{~s}$, the count rate of the PC data becomes weak due to the rapid temporal decline and we have only upper limits (see Fig. 2). All the PC data were taken after the two flares described in the previous section. However, the data we have for the first flare were successively taken in IM, PuPD and LrPD modes. We do not have enough counts in the photo-diode modes to properly extract a spectrum, therefore no spectral information could be determined for this flare.

All the spectra were binned at $15 \mathrm{cts}^{\mathrm{bin}}{ }^{-1}$ within XSPEC v11.3.1 (Arnaud 1996). The WT mode spectrum and the "early"

2 The five fainter bursts in this sample are 2 short bursts (GRB 050509b, Gehrels et al. 2005; GRB 050813, Fox et al. 2005), GRB 050815, the XRF 050406 (Romano et al. 2006) and GRB 050223 (Page et al. 2005). and "late" PC mode spectra were fit by an absorbed power-law taking an excess absorption column (defined in the observed frame) $\Delta N_{\mathrm{H}}$ into account. We used two photo-electric absorption (PHABS) models within XSPEC: one fixed to the value of the Galactic absorption column and the other kept free.

The fitted parameters for the WT spectrum are given in Table 2 as case 2 . Note that the WT spectrum included the second X-ray flare. We also divided the WT data into two segments, to look for spectral softening during the flare, as reported by Burrows et al. (2005b): obtaining one spectrum before the second flare (bf) with an exposure of $27 \mathrm{~s}$, and one containing the flare (f) with an exposure of $29 \mathrm{~s}$. The fits suggest that the spectral slope of the flare $\left(\beta_{\mathrm{f}}=0.4 \pm 0.3\right)$ is steeper than that of the WT data before the flare $\left(\beta_{\mathrm{bf}}=0.0 \pm 0.3\right)$ using a fixed value of $\Delta N_{\mathrm{H}}=4.4 \times 10^{21} \mathrm{~cm}^{-2}$. However, the large errors in the slopes do not require a spectral variation.

To determine whether the early X-ray emission is a continuation of the Gamma-ray emission, we fit the BAT and WT spectra (see Fig. 3) with a single absorbed power-law, but with different normalisation to take into account the temporal decay between the two spectra. We fixed the $\Delta N_{\mathrm{H}}$ value to that found for the WT spectra. A single absorbed power-law gives a good fit (see case 3 in Table 2), consistent with the hypothesis that the X-ray and Gamma-ray emissions were produced by the same emission mechanism.

The parameters of the PC spectra are not well constrained using the $\chi^{2}$ statistic, due to the low number of counts in the spectra (less than 80 counts). Therefore, we fit the "early" and "late" PC spectra using the Cash statistic (Cash 1979). Both PC spectra were binned to $1 \mathrm{cts} \mathrm{bin}^{-1}$. The results are summarised in Table 2 as case $4 a$. Note that the parameter uncertainties are large due to the low statistics in the PC spectra. 
Table 2. Summary of the Gamma-ray and X-ray spectral fitting parameters.

\begin{tabular}{cccccc}
\hline \hline Case & Spectrum & $\Delta N_{\mathrm{H}}(z=0)\left(\times 10^{21} \mathrm{~cm}^{-2}\right)$ & $\beta$ & $F_{2-10 \mathrm{keV}}^{a}$ & $\chi^{2}$ (d.o.f.) \\
\hline 1 & BAT & - & $0.7 \pm 0.5$ & - & $54(56)$ \\
\hline 2 & WT & $4.4_{-3.5}^{+4.0}$ & $0.1 \pm 0.3^{d}$ & $3.1_{-0.8}^{+0.5} \times 10^{-10}$ & $13(18)$ \\
\hline 3 & BAT-XRT/WT & 4.4 fixed & $0.2 \pm 0.2$ & & $57(76)$ \\
\hline 4a & PC "early" & $6.9_{-3.2}^{+5.9}$ & $1.0_{-0.8}^{+0.5}$ & $7.6_{-2.7}^{+1.4} \times 10^{-11}$ & $167^{b}(174)^{c}$ \\
4a & PC "late" & $3.8_{-3.2}^{+3.6}$ & $1.5_{-0.7}^{+0.8}$ & $4.9_{-2.2}^{+1.6} \times 10^{-12}$ & $167^{b}(174)^{c}$ \\
\hline 4b & PC "early" & 4.4 fixed & $1.2_{-0.1}^{+0.3 d}$ & $5.9_{-1.2}^{+2.0} \times 10^{-11}$ & $148^{b}(174)^{c}$ \\
4b & PC "late" & 4.4 fixed & $1.2_{-0.1}^{+0.3 d}$ & $6.9_{-2.1}^{+2.5} \times 10^{-12}$ & $148^{b}(174)^{c}$ \\
\hline
\end{tabular}

${ }^{a}$ Observed fluxes in units of erg $\mathrm{cm}^{-2} \mathrm{~s}^{-1} \cdot{ }^{b}$ Cash statistic value. ${ }^{c}$ PHA bins. ${ }^{d}$ The uncertainties at a $99 \%$ confidence level for one parameter of interest $\left(\Delta \chi^{2}=6.63\right)$ are $\beta=0.1_{-0.5}^{+0.6}$ and $\beta=1.2_{-0.3}^{+0.5}$ respectively for WT and PC spectra.

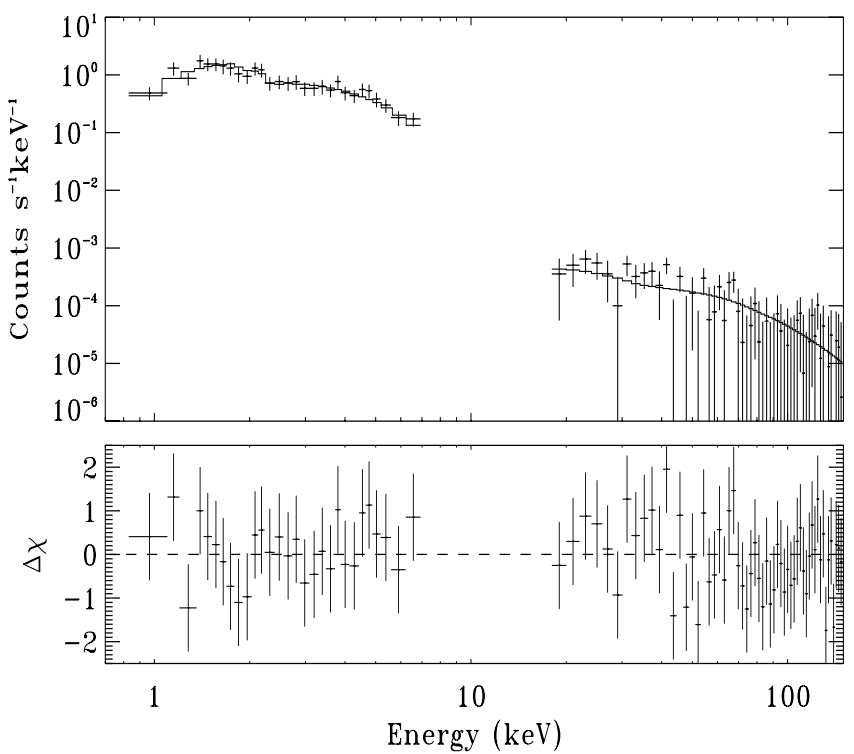

Fig. 3. Spectra from the BAT (on the right) and the WT data (on the left) fit together with an absorbed power-law $(\beta=0.2 \pm 0.2)$.

To better constrain the errors, we fit the PC spectra this time assuming the same spectral index $\beta$ and $\Delta N_{\mathrm{H}}$ values. Indeed, the $\beta$ and $\Delta N_{\mathrm{H}}$ values given in case $4 \mathrm{a}$ in Table 2 are consistent. We found a value of $\Delta N_{\mathrm{H}}=4.0_{-1.2}^{+1.0} \times 10^{21} \mathrm{~cm}^{-2}$, consistent with that found for the WT spectrum. We then fixed the value of $\Delta N_{\mathrm{H}}=$ $4.4 \times 10^{21} \mathrm{~cm}^{-2}$ and estimated the uncertainties on the $\beta$ spectral slopes (see case $4 \mathrm{~b}$ in Table 2 ).

The values of the excess absorption column would be higher in the rest frame of the burst. We tried to constrain the redshift and $\Delta N_{\mathrm{H}}$ in the rest frame of the burst using the absorption models taking into account the redshift within XSPEC. However, no useful constraints on these two parameters could be found.

From the best fits shown in Table 2 (cases 2 and $4 b$ ), it appears that GRB 050421 softens between the two time intervals $T_{0}+115 \rightarrow 171 \mathrm{~s}$ and $T_{0}+171 \rightarrow 771 \mathrm{~s}$. If the spectral softening is real, the spectral evolution seen in our data could be due to a shift of the peak of the prompt emission down to the lower energies (Ford et al. 1995).

\subsection{Combined Gamma-ray and X-ray light-curve}

We used the slope of the joint BAT and XRT/WT spectra to extrapolate the BAT light-curve into the XRT $0.3-10 \mathrm{keV}$

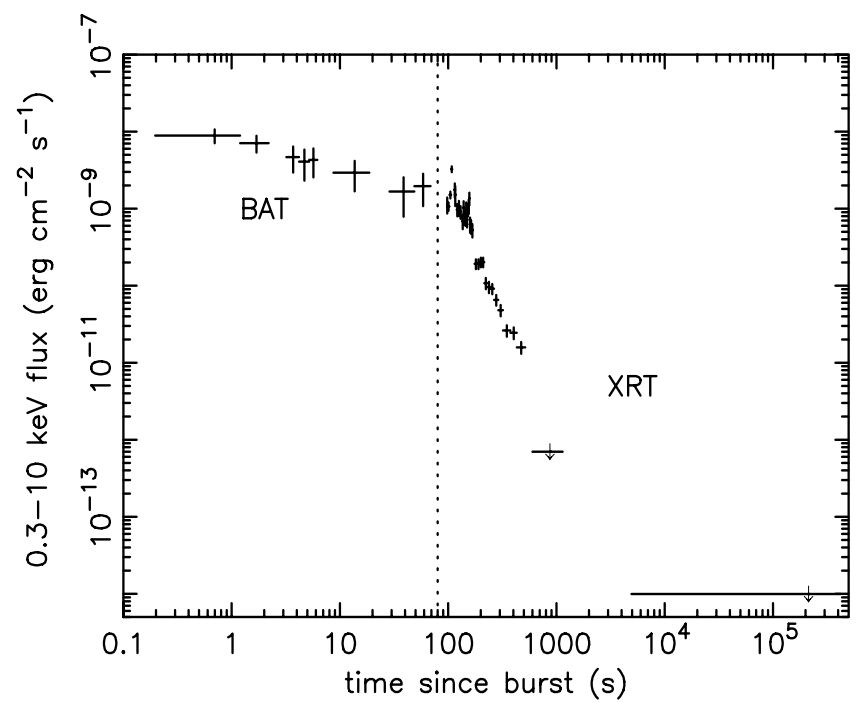

Fig. 4. Combined light-curves of the BAT data extrapolated in the 0.3-10 keV energy band and the XRT data in the same band. The dotted vertical line indicates the separation between the BAT and XRT data. The unabsorbed fluxes are given in units of erg cm $\mathrm{cm}^{-2} \mathrm{~s}^{-1}$. The upper limits are given at $3 \sigma$.

energy range. Figure 4 shows the BAT-XRT light-curve (in units of erg cm $\mathrm{cm}^{-2} \mathrm{~s}^{-1}$ ). For the first flare, peaking at 110 seconds, we supposed what its spectral slope is the same as that for the combined XRT-BAT spectrum.

Note that the end of the BAT light-curve matches well with the beginning of the XRT light curve. The time of the transition from the gamma-ray emission to the early X-ray decay is $\sim 9-11 \times T_{90}$. From the first LrPD data, it is not completely clear to see if the decay start before or after the first flare. The $T_{90}$ value found for this burst could then underestimate the real duration of this burst, due to skew in instrument sensitivity.

\subsection{Energy of the $X$-ray flares}

It is difficult to estimate the fluence of the first flare, because its shape is poorly known due to the lack of data (see Sect. 3.1.2). Using the XRT/BAT spectral index $(\beta \sim 0.2)$ and assuming a Gaussian shape for the flare, we found a fluence of $3.1_{-1.5}^{+5.2} \times$ $10^{-8} \mathrm{erg} \mathrm{cm}^{-2}$ in the XRT energy band, which corresponds to $20-75 \%$ of the GRB fluence in the $15-150 \mathrm{keV}$ band.

During the second flare, the fluence in the $0.3-10 \mathrm{keV}$ band was $6.1_{-2.7}^{+4.9} \times 10^{-9} \mathrm{erg} \mathrm{cm}^{-2}$ (subtracting the underlying 
power-law light-curve). This fluence corresponds to $\sim 3-10 \%$ of the total fluence of the burst in the $15-150 \mathrm{keV}$ band.

\section{Discussion}

\subsection{Summary of the main results}

The main results of the BAT and XRT temporal and spectral analysis of GRB 050421 are:

- The BAT light-curve showed one FRED peak with a duration of $T_{90} \sim 10.3 \mathrm{~s}$ and a tail extending to $\sim T_{0}+70 \mathrm{~s}$. The burst is faint, with a fluence of $1.1 \pm 0.7 \times 10^{-7} \mathrm{erg} \mathrm{cm}^{-2}$ in the $15-$ $150 \mathrm{keV}$ band. GRB 050421 is in the lowest $10 \%$ of GRBs detected by the BAT in term of fluence.

- The global XRT light-curve showed a rapid decline of the early X-ray emission $(\alpha=3.1 \pm 0.1)$. Due to the rapid decline, the X-ray emission was no longer detected beyond $1 \mathrm{~h}$. The $3 \sigma$ upper limit on the unabsorbed flux in the $0.3-10 \mathrm{keV}$ band is $7 \times 10^{-13} \mathrm{erg} \mathrm{cm}^{-2} \mathrm{~s}^{-1}$ at $\sim T_{0}+900 \mathrm{~s}$. The XRT lightcurve also showed at least two flares peaking at $T_{0}+110 \mathrm{~s}$ and $T_{0}+154 \mathrm{~s}$.

- There is a possible spectral softening from the early $T_{0}+$ $115-T_{0}+171 \mathrm{~s}$ data with $\beta=0.1 \pm 0.3$ to the later $\sim T_{0}+$ $180-T_{0}+771$ s data with $\beta=1.2_{-0.1}^{+0.3}$.

- The joint fit of the BAT and early XRT data shows that they are likely to be produced by the same emission mechanism, which is consistent with an absorbed single power-law with a $\beta \sim 0.2$ slope.

- Excess absorption, $\Delta N_{\mathrm{H}}(z=0) \sim 4.4 \times 10^{21} \mathrm{~cm}^{-2}$, over that due to our Galaxy is seen in the XRT spectra. No variation in absorption with time is seen.

\subsection{The rapid decline of the $X$-ray emission}

The rapid decline of the X-ray emission is unlikely to be produced by the emission from the forward shock. Indeed, the relations between $\alpha$ and $\beta$ for a forward shock emission in the ISM and wind afterglow models, even taking the energy injection into account, are incompatible with the spectral and temporal characteristics of this burst (see Table 2 in Zhang et al. 2006).

It is interesting to consider the "high latitude emission" model (Kumar \& Panaitescu 2000; Dermer 2004; Zhang et al. 2006). In this model, the tail of the prompt emission is produced at high angles relative to the observer direction (with angles $\theta>\Gamma^{-1}$ ). This radiation takes longer to reach the observer due to the curvature of the emitting shell. The curvature relation $\alpha=2+\beta$ can well explain the temporal decline we see after $\sim T_{0}+180 \mathrm{~s}$, since we found $\alpha=3.1 \pm 0.1$ and $\beta_{\mathrm{PC}}=1.2_{-0.1}^{+0.3}$, which are consistent with the curvature relation. Moreover, using the $\alpha=2+\beta$ relation and the XRT/WT spectral slope $(\beta=0.1 \pm 0.3)$, we have $\alpha=2.1 \pm 0.3$. This value of the decay slope is consistent with that derived in Sect. 3.1.2 from the fit of the WT part of the light-curve before $T_{0}+180 \mathrm{~s}(\alpha=2.0 \pm 0.6)$.

If the later temporal decay seen in several XRT bursts is linked to the forward shock emission (Nousek et al. 2006), then the transition between the tail of the prompt emission and the forward shock emission is not seen in our data. If a forward shock has been produced, then its emission would be very faint and below the XRT sensitivity threshold. A possible and simple explanation would be that the very local surrounding density is so low that the blastwave did not sweep up sufficient matter to produce a sufficiently bright afterglow.
Note that to date (before the end of September 2005) only 8 GRBs detected by the BAT (GRB 050416b, GRB 050418, GRB 050507, GRB 050502a, GRB 050709, GRB 050715, GRB 050911 and GRB 050925) were not detected by the XRT. In each case this was because the spacecraft did not slew or the slew was very delayed due to an observing constraint. Several authors have reported that the steep decline of the early part of the X-ray light-curve is due to high latitude emission e.g. GRB 050117a (Hill et al. 2005a) and GRB 050315 (Vaughan et al. 2006). Contrary to GRB 050421, the steep decline seen in GRBs 050117 and 050315 was followed by a shallower decline, and later by another steep decline. Thus, GRB 050421 appears unique so far in apparently not showing emission from the forward shock.

\subsection{The $X$-ray flares as a signature of a late activity of the central engine?}

The short timescales $\delta t / t_{\text {peak }} \ll 1$ for the two flares, the large flux variations $\left(\delta F / F_{\text {peak }} \sim 3.7\right)$ for the first flare, and the fact that the two flares would reasonably be interpreted by a model with the same underlying physics rule out most of the models based on the external shocks (Zhang et al. 2006). Moreover, Ioka et al. (2005) have shown that simple kinematic arguments can give limits on the timescale and amplitude variabilities in the Xray afterglows for different cases. The timescale and amplitude variabilities for the two X-ray flares seen in GRB 050421 are located in the upper part of their Fig. 1, and therefore violate the cases b (ambient density fluctuations) and c (patchy shells) defined in Ioka et al. (2005).

When a blastwave is slowed down by the external medium, forward and reverse shocks are expected to be produced. Usually, optical, UV and radio emission are expected when the reverse shock crosses the external medium (e.g. Kulkarni et al.; Akerlof et al. 1999). Kobayashi et al. (2005) have shown that a strong flare could be produced in the X-ray band, if Synchrotron Self Compton (SSC) emission is invoked. This case would require some carefully balanced conditions. Even so it is still difficult to explain the rising and decaying slopes of the first flare, and how the reverse shocks could produce the two flares.

An alternative is then to invoke internal shocks. Assume that the central engine emits, in a single event, matter with a range of Lorentz factors, $\Gamma$. When the lower $\Gamma$ matter catches up with the decelerated high $\Gamma$ matter, the lower $\Gamma$ matter would inject additional energy in the blastwave and a flare could be produced (Ramirez-Ruiz et al. 2002). The decaying timescale ratio in this model is $\delta t_{\text {decay }} / t_{\text {peak }} \sim 1$ and the flux level does not resume the previous level due to the additional energy injected (Zhang et al. 2006). Both of these predicted behaviours are inconsistent with our data. Moreover, the timescale and amplitude variabilities for the two X-ray flares violate the case d (refreshed shocks) defined in Ioka et al. (2005).

A more likely possibility is that the two X-ray flares are produced by late internal shocks. This explanation has already been invoked to explain the X-ray flares in several papers (e.g. Burrows et al. 2005; Falcone at al. 2006; King et al. 2005; Romano et al. 2006). The two X-ray flares of GRB 050421 are within the known range of the GRB durations (Paciesas et al. 1999).

\section{Conclusion}

To date, GRB 050421 was one of the faintest bursts observed by Swift. It was only observed in the X-ray and Gamma-ray 
bands. It had a rapid temporal decline of the X-ray emission on which were superposed at least two X-ray flares. We have argued that the flares are probably produced by late internal shocks. The spectral softening seen in the X-ray data is likely due to the shift of the peak of the prompt spectrum in the XRT energy band. We have argued that the temporal decline corresponds to the tail of the prompt emission, which is produced by high latitude emission. Beyond $1 \mathrm{~h}$ after the BAT trigger, the source was undetectable. The $3 \sigma$ upper limit on the unabsorbed flux in the $0.3-10 \mathrm{keV}$ band is $<7 \times 10^{-13} \mathrm{erg} \mathrm{cm}^{-2} \mathrm{~s}^{-1}$ at $T_{0}+900 \mathrm{~s}$.

If the explanation above is correct, then GRB 050421 would be the first burst to which the XRT slewed promptly, for which no forward shock was observed. Therefore, GRB 050421 would be the first "naked burst" detected by Swift.

Acknowledgements. O.G., K.L.P., M.R.G., A.P.B., J.P.O. gratefully acknowledge PPARC funding. This work issupported at OAB by ASI grant I/R/039/04 and at Penn State by NASA contract NAS5-0136.

\section{References}

Akerlof, C., Balsano, R., Barthelemy, S., et al. 1999, Nature, 398, 400 Arnaud, K. 1996, in Astronomical Data Analysis Software and Systems, ed. J. G. Barnes., ASP Conf. Ser., 101, 17

Band, D., Matteson, J., Ford, L., et al. 1993, ApJ, 413, 281

Barbier, L. M. et al. 2005, GCN Circular 3296

Barthelmy, S., Barbier, L. M., Cummings, J. R., et al. 2005, Space Sci. Rev., 120, 143

Bloom, J. S., et al. 2005, GCN Circ. 3306

Blustin, A., et al. 2005, GCN Circ. 3307

Burrows, D. N., Hill, J. E., Nousek, J. A., et al., 2005a, Space Sci. Rev., 120, 165

Burrows, D. N., Romano, P., Falcone, A. D., et al. 2005b, Science, 309, 1833

Cash, W. 1979, ApJ, 228, 939

Davies, M. B., King, A., Rosswog, S., \& Wynn, G. 2002, ApJ, 579, 63

Dermer, C. 2004, ApJ, 614, 284

Dezalay, J. P., Lestrade, J. P., \& Barat, C. 1996, ApJ, 471, 27
Dickey, J. M., \& Lockman, F. J. 1990, ARA\&A, 28, 215

Falcone, A. D., Burrows, D. N., Lazzati, D., et al. 2006, ApJ, in press

Ford, L. A., Band, D. L., Matteson, J. L., et al. 1995, ApJ, 439, 307

Gehrels, N., Sarazin, C. L., O'Brien, P. T., et al. 2005, Nature, 437, 851

Gehrels, N., Chincarini, G., Giommi, P., et al. 2004, ApJ, 611, 1005

Godet, O., et al. 2005 GCN Circular 3301

Hill, J. E., Burrows, D. N., Nousek, J. A., et al. 2004, X-Ray and GammaRay Instrumentation for Astronomy XIII, ed. K. A. Flanagan, O. H. W. Siegmund, Proc. SPIE, 5165, 217

Hill, J. E., Morris, D. C., Sakamoto, T., et al. 2005a, ApJ, in press, [arXiv: astro-ph/0510008]

Hill, J. E., Angelini, L., Morris, D. C., et al. 2005b, Proc. SPIE, 5898, 325

Ioka, K., Kobayashi, S., \& Zhang, B. 2005, ApJ, 631, 429

Jelinek, M., et al. 2005, GCN Circ. 3298

King, A., O'Brien, P. T., Goad, M. R., et al. 2005, ApJ, 630, 113

Kobayashi, S., Piran, T., \& Sari, R. 1997, MNRAS, 490, 92

Kobayashi, S., Zhang, B., Mészáros, P., \& Burrows, D. N. 2005, ApJ, [arXiv: astro-ph/0506157]

Kouveliotou, C., Meegan, C. A., Fishman, G. J., et al. 1993, ApJ, 413, 101

Kulkarni, S. R., Frail, D. A., Sari, R., et al. 1999, ApJ, 522, 97

Kumar, P., \& Panaitescu, A. 2000, ApJ, 541, 51

Mészáros, P. 2000, Nucl. Phys. B, 80, 63

Mészáros, P., \& Rees, M. J. 1993, ApJ, 405, 278

Moretti, A., Perri, M., Capalbi, M., et al. 2006, A\&A, 448, L9

Nousek, J. A., Kouveliotou, C., Grupe, D., et al. 2006, ApJ, in press, [arXiv: astro-ph/0508332]

O'Brien, P. T., et al. 2006, ApJ, submitted

Paciesas, W. S., Meegan, C. A., Pendleton, G. N., et al. 1999, ApJS, 122, 465

Page, K. L., Rol, E., Levan A. J., et al. 2005, MNRAS, 363, 76

Panaitescu, A., \& Mészáros, P. 1998, ApJ, 492, 683

Piro, L., De Pasquale, M., Soffitta, P., et al. 2005, ApJ, 623, 314

Ramirez-Ruiz, E., Celotti, A., \& Rees, M. J. 2002, MNRAS, 337, 1349

Rees, M. J., \& Mészáros, P. 1994, ApJ, 430, 93

Romano, P., Moretti, A., Banat, A., et al. 2006, A\&A, in press

Roming, P. W. A., Kennedy, T. E., Mason, K. O., et al. 2005, Space Sci. Rev., 120,95 ,

Sari, R., \& Piran, T. 1997, ApJ, 485, 270

Sakamoto, T., et al. 2005, GCN Circular 3305

Tagliaferri, G., Goad, M. R., Chincarini, G., et al. 2005, Nature, 436, 985

Vaughan, S., Goad, M. R., Beardmore, A. P., et al. 2006, ApJ, in press, [arXiv: astro-ph/0510677]

Zhang, B., \& Mészáros, P. 2004, Int. J. Mod. Phys. A, 19, 2385

Zhang, B., Zhong Fan, Y., Dyks, J., et al. 2006, ApJ, in press, [arXiv: astro-ph/0508321] 